\title{
IN-PLANE VIBRATIONS OF ANNULAR RINGS
}

\author{
G. Ambati, J. F. W. Bell and J. C. K. Sharp \\ Department of Electrical Engineering, \\ The University of Aston in Birmingham, Birmingham B4 7PB, England
}

(Received 9 October 1975, and in revised form 18 March 1976)

\begin{abstract}
The in-plane vibrations of thin disks and narrow thin rings which already have been completely analysed are the two extremes of the annulus configuration. The general case of the annulus has now been analysed and the results confirmed by experiment.

The modes of disks are characterized by two integers giving the number of nodal circles -including a possible central nodal point - - and the number of nodal diameters. In the absence of nodal diameters two series of pure radial and pure tangential modes are possible. These have the characteristics of plate and shear waves respectively. Compound modes which have both radial and tangential components of displacement have been found to fall into four modal series.

There are six series of narrow ring counterparts of these disk resonances, every disk mode moving to a particular ring mode as the hole is developed in the centre. Flexural modes approach zero frequency, the extensional series have finite frequencies and there is one series of compound shear modes and one of compound plate modes both of which approach infinite frequency as do the two pure modes. The eigenvalues, in the form of a dimensionless frequency constant, have been evaluated for steps of hole size from the disk case to that of an indefinitely narrow ring and for a wide range of values of Poisson's ratio. The theoretical results have been supported by measurements of the spectra of a range of annuli and by exploring the pattern of vibrations with a probe pick-up.

A feature of the change from disk to thin ring is the disappearance of one or more nodal circles in certain cases. Typically all modes with one nodal circle and two or more nodal diameters lose the nodal circle to become the finite frequency extensional ring series. Compound modes having $2,4, \ldots$ circles as disks have $1,2, \ldots$ circles as shear ring modes and the $3,5, \ldots$ series become $1,2, \ldots$ plate ring modes. This creates the apparent paradox that the inner free boundary of an annulus can be a node. In fact the force at the edge arising from a radial strain is balanced by the hoop stress arising from a tangential strain.

The parallel between the modes of resonance of thin rings and infinite tubes is drawn.
\end{abstract}

\section{INTRODUCTION}

The vibrations of solid bodies have been solved for only a few simple geometries and modes. In the cases where a single wave equation is involved straightforward algebraic solutions for the resonant frequencies were obtained in the nineteenth century. Typically, for thin narrow annular rings Hoppe [1] in 1871 established the frequency spectrum of flexural vibrations in which flexural waves travel round the ring and later, in 1889, Rayleigh [2] found the finite frequency spectrum involving extensional (rod) waves. In the case of thin disks the in-plane vibrations are compounded of radial and torsional waves and each problem, separately, can be readily solved. When these two types of vibration occur together the simultaneous solution of both wave equations is necessary and the results, which in the decoupled state are reasonably simple functions of plate and shear velocities, respectively, now have an additional dependence on Poisson's ratio which varies from mode to mode. In 1966 Holland [3], making extensive use of digital computation, obtained accurate numerical solutions for a comprehensive range of compound modes, and Poisson's ratio values ranging from 0.25 to 0.50 , covering the commoner engineering materials. 
In parallel with this development the study of the propagation of waves in infinite cylindrical rods, initiated by Pochammer and Chree, was proceeding. Where in the case of the disk the thin condition simplifies the vibrations because of the direct relationship between radial and thickness strain, in the case of the cylinder the infinite condition simplifies the axial movement to plane strain propagation. In both cases only the boundary conditions at the cylindrical surface are necessary. Where the equations for disks involve shear and plate waves in cylinders the corresponding waves are those of shear and bulk.

A study of the work of Holland indicated that the general case of a thin annular ring, an important engineering component introducing a second boundary condition, while increasing the number of equations from four to sixteen, would yield a usefully large number of solutions at reasonable cost in computer time. To include the graphites and ceramics as well as the glasses, metals and alloys, the mode frequencies were calculated for Poisson's ratio ranging from 0 to 0.5 in steps of $0 \cdot 01$.

These results identify the disk counterparts of the ring modes and vice versa. Typically the finite frequency extensional ring mode series must go to a disk series of which the pure radial mode is the first but the counterparts of the higher modes are not self-evident. The results also provide practically useful data on the effect of a small hole in a disk or on a finite width ring, hitherto only available as approximations.

\section{TABLE 1}

Classification of disk modes

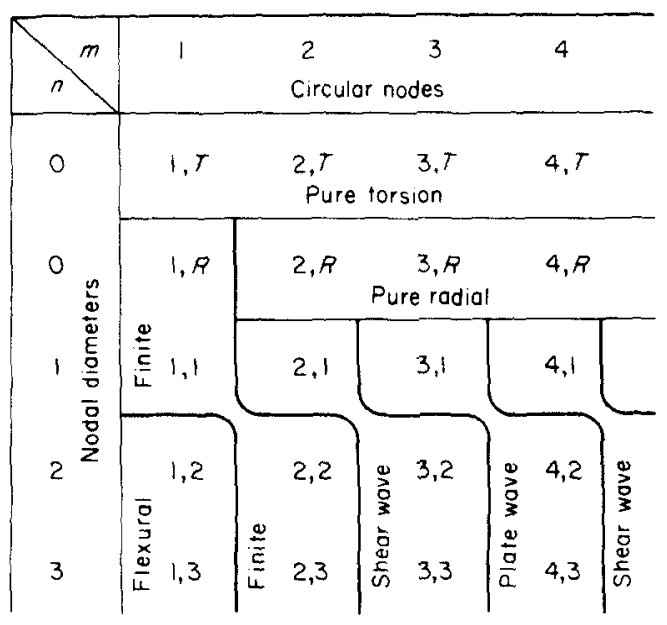

Table 1 shows a classification of disk modes defined by $(m, n)$ where $m$ is the number of nodal circles-a centre nodal point being included-and $n$ is the number of nodal diameters. There are two series with no nodal diameters, the tangential modes where the first $(1, T)$ has one nodal circle and the movement is purely rotational, and the radial modes where the first $(1, R)$ has a node at the centre and the movement is purely radial. Thus $(1, T)$ and $(2, R)$ are equivalents, there being no tangential equivalent to $(1, R)$.

Resonant frequencies are expressed as a dimensionless scaling constant $K$ where

$$
K=\omega \times(\text { a dimension }) /(\text { a velocity }) \text {. }
$$

This follows Rayleigh's Principle of Similitude, as a scale increase by a factor will decrease the frequency by the same factor. In thin rings and disks the frequency is independent of thickness and consequently the radius is normally taken as the dimension. In the case of annular rings, by taking the width as the dimension the modes which move to infinite narrow 
TABLE 2

Characteristics of disk resonances

\begin{tabular}{|c|c|c|c|}
\hline Mode series & Typical nodal patterns & Remarks & Source of frequency term \\
\hline $\begin{array}{l}\text { Pure torsional } \\
\text { disk }\end{array}$ & Drive & $\begin{array}{l}\text { The dynamic balance con- } \\
\text { dition requires at least } \\
\text { one nodal circle. The } \\
\text { centre is always an anti- } \\
\text { node. This series consists } \\
\text { of }(1, T) ;(2, T) ;(3, T) ; \ldots\end{array}$ & $\begin{array}{l}\text { Solution of equation } \\
K_{s} \mathrm{~J}_{0}\left(K_{s}\right) / \mathrm{J}_{1}\left(K_{s}\right)=2 \text {, } \\
\text { where } K_{s}=\omega a / C_{s} \text {. For- } \\
\text { mal solution by Love }\end{array}$ \\
\hline $\begin{array}{l}\text { Pure radial } \\
\text { disk }\end{array}$ & Mode I & $\begin{array}{l}\text { The dynamic balance con- } \\
\text { dition does not require a } \\
\text { nodal circle. The centrc is } \\
\text { always a node. This } \\
\text { series consists of }(1, R) \text {; } \\
(2, R) ;(3, R) ; \ldots\end{array}$ & $\begin{array}{l}\text { Solution of equation } \\
K_{p} \mathrm{~J}_{0}\left(K_{p}\right) / \mathrm{J}_{1}\left(K_{p}\right)=1-\sigma, \\
\text { where } K_{p}=\omega a / C_{p} . \\
\text { Normally attributed to } \\
\text { S. E. Poisson. Formal } \\
\text { solution by Love }\end{array}$ \\
\hline $\begin{array}{l}\text { Compound } \\
\text { disk }\end{array}$ & & $\begin{array}{l}\text { This series consists of }(1,1) \\
\text { and higher order modes }\end{array}$ & $\begin{array}{l}\text { Solution of equation (16) } \\
\quad \text { where } K_{s}=\omega a / C_{s}, K_{p}= \\
\omega a / C_{p}, K_{0}=\omega a / C_{0} . M . \\
\text { Onoe and R. Holland }\end{array}$ \\
\hline
\end{tabular}

TABLE 3

Characteristics of narrow ring resonances

Mode series Typical nodal pattern
Extensional


ring frequency approach a constant $K$ value. Similarly for the modes which move to zero frequency the simplest presentation of results is obtained by taking (mean radius) ${ }^{2} /$ width. The theoretical results usually appear in terms of plate or shear velocities. By choosing an appropriate velocity the $K$ value can be made independent of Poisson's ratio for a particular series of modes as the thin ring condition is approached. Rod, plate and shear velocities are appropriate for extensional, radial and tangential modes respectively.

Tables 2 and 3 summarize the main features of disk and narrow ring modes and include the frequency equations. Where the motion is predominantly radial, a radial drive is used and tangential vibrations require an angled drive. The drive point normally becomes an antinode for the drive direction. Nodal lines refer to radial or tangential movements. In general a node for one component of motion is an antinode for its orthogonal counterpart. The phase of the various regions is indicated by arrows or polarity signs.

\section{THEORETICAL ANALYSIS}

In the analysis of a resonant system, differential equations for the various wave motions are first established in the appropriate co-ordinates, cylindrical in this case. A general solution of these wave equations must then be found. The boundary conditions for the system, in this case that the radial and tangential stresses must vanish at the inner and outer perimeters, are then used to obtain the specific solution. This will consist of simultaneous algebraic equations containing amplitude constants and the eigenvalue $(K)$ giving the frequency in terms of the dimensions of the resonator and a wave velocity. There will be an infinite number of numerical solutions, each corresponding to a mode of vibration. If required, the amplitude constants can also be evaluated to obtain the eigenfunction of the mode expressing the components of stress or strain throughout the resonator. Aspects of the form of the function, in the disk case the numbers of nodal circles and diameters, $m$ and $n$, are used to classify the modes.

The wave equations are conveniently derived by Love [4], the strains being rotational, $\bar{\omega}$, and dilatational, $\Delta$, which are related to the tangential and radial displacements $\xi_{\theta}$ and $\xi_{r}$, respectively, by (a list of symbols is given in the Appendix)

$$
\begin{gathered}
2 \bar{\omega}=\partial \xi_{\theta} / \partial r+(1 / r)\left(\xi_{\theta}+\partial \xi_{r} / \partial \theta\right), \\
\Delta=\partial \xi_{r} / \partial r+(1 / r)\left(\xi_{r}+\partial \xi_{\theta} / \partial \theta\right) .
\end{gathered}
$$

The wave equations are, for shear and plate waves,

$$
\begin{aligned}
& \nabla^{2} \bar{\omega}=\left(1 / C_{s}^{2}\right) \partial^{2} \bar{\omega} / \partial t^{2}, \\
& \nabla^{2} \Delta=\left(1 / C_{p}^{2}\right) \partial^{2} \Delta / \partial t^{2} .
\end{aligned}
$$

It is worthy of note here that the infinite shell wave equations have the same forms as equations (4) and (5) with the dilatational velocity replacing that of the plate. The Laplace expressions involve additional longitudinal terms associated with wave propagation. These are omitted for the cut-off frequency (no propagation) analysis. In this case the frequency equation is a $4 \times 4$ determinant similar to equation (12). Armenakas, Gazis and Herrmann [5] have given numerical solutions (restricted to a single value of Poisson's ratio) and these are used below in comparing resonances of the two geometrical forms.

The algebraic solutions of the wave equations are given by

$$
\begin{aligned}
& \Delta=\left[A_{1} \mathrm{~J}_{n}(h r)+A_{2} \mathrm{Y}_{n}(h r)\right] \cos n \theta \cos \omega t, \\
& \bar{\omega}=\left[A_{3} \mathrm{~J}_{n}(k r)+A_{4} \mathrm{Y}_{n}(k r)\right] \sin n \theta \cos \omega t,
\end{aligned}
$$


where $h=\omega / C_{p}, k=\omega / C_{s}$ and $A_{1}, A_{2}, A_{3}$ and $A_{4}$ are the amplitude constants.

These equations can be rewritten to give expressions for. $\xi_{r}$ and $\xi_{\theta}$ :

$$
\begin{aligned}
& \xi_{r}=\left[A_{5} \frac{\partial}{\partial r} \mathrm{~J}_{n}(h r)+\frac{n A_{6}}{r} \mathrm{~J}_{n}(k r)+A_{7} \frac{\partial}{\partial r} \mathrm{Y}_{n}(h r)+\frac{n A_{8}}{r} \mathrm{Y}_{n}(k r)\right] \cos n \theta \sin \omega t \\
& \xi_{\theta}=\left[\frac{n A_{5}}{r} \mathrm{~J}_{n}(h r)+A_{6} \frac{\partial}{\partial r} \mathrm{~J}_{n}(8 r)+\frac{n A_{7}}{r} \mathrm{Y}_{n}(h r)+A_{8} \frac{\partial}{\partial r} \mathrm{Y}_{n}(k r)\right] \sin n \theta \sin \omega t
\end{aligned}
$$

where $A_{5}, A_{6}, A_{7}$ and $A_{8}$ are also amplitude constants. The two boundary conditions for the annulus are

$$
\begin{aligned}
& \text { radial stress resultant }=\text { plate modulus }\left[\frac{\partial \xi_{r}}{\partial r}+\frac{5}{r}\left(\xi_{r}+\frac{\partial \xi_{\theta}}{\partial \theta}\right)\right], \\
& \text { tangential stress resultant }=\text { shear modulus }\left[\frac{\partial \xi_{\theta}}{\partial r}-\frac{1}{r}\left(\xi_{\theta}-\frac{\partial \xi_{r}}{\partial r}\right)\right] .
\end{aligned}
$$

Modes of vibration can consist of purely torsional or purely radial vibrations in which cases the displacements are independent of $\theta$ and $n=0$, or compound modes in which both components are present and are dependent on $\theta, n$ being one or more. In the case of pure radial modes equation (8) is zero, the $A_{6}$ and $A_{8}$ terms of equation (8) are zero and the boundary condition given by equation (10) has no $\xi_{\theta}$ term. The torsional case involves equations (8) and (9) in a similar way. The frequency equations obtained for these two special cases, as for the annular ring, are the $2 \times 2$ determinants considered below. The general case is the compound mode for which the frequency equation is a $4 \times 4$ determinant and the computer program solving it numerically has been used for all calculations. Equation (12) gives the determinant and the expressions for its terms:

where

$$
f(K)=\left|\begin{array}{llll}
a_{11} & a_{12} & a_{13} & a_{14} \\
a_{21} & a_{22} & a_{23} & a_{24} \\
a_{31} & a_{32} & a_{33} & a_{34} \\
a_{41} & a_{42} & a_{43} & a_{44}
\end{array}\right|=0
$$

$$
\begin{array}{ll}
a_{11}=-\mathrm{J}_{n}(K)\left[(K \Theta)^{2} / 2-n(n+1)+\mathrm{M}_{n}(K)\right], & a_{12}=n \mathrm{~J}_{n}(K \Theta)\left[\mathrm{M}_{n}(K \Theta)-(n+1)\right], \\
a_{13}=-\mathrm{Y}_{n}(K)\left[(K \Theta)^{2} / 2-n(n+1)+\mathrm{N}_{n}(K)\right], & a_{14}=n \mathrm{Y}_{n}(K \Theta)\left[\mathrm{N}_{n}(K \Theta)-(n+1)\right], \\
a_{21}=-\mathrm{J}_{n}(L)\left[(L \Theta)^{2} / 2-n(n+1)+\mathrm{M}_{n}(L)\right], & a_{22}=n \mathrm{~J}_{n}(L \Theta)\left[\mathrm{M}_{n}(L \Theta)-(n+1)\right], \\
a_{23}=-\mathrm{Y}_{n}(L)\left[(L \Theta)^{2} / 2-n(n+1)+\mathrm{N}_{n}(L)\right], & a_{24}=n \mathrm{Y}_{n}(L \Theta)\left[\mathrm{N}_{n}(L \Theta)-(n+1)\right], \\
a_{31}=-n \mathrm{~J}_{n}(K)\left[\mathrm{M}_{n}(K)-(n+1)\right], & a_{32}=\mathrm{J}_{n}(K \Theta)\left[(K \Theta)^{2} / 2-n(n+1)+\mathrm{M}_{n}(K \Theta)\right], \\
a_{33}=-n \mathrm{Y}_{n}(K)\left[\mathrm{N}_{n}(K)-(n+1)\right], & a_{34}=\mathrm{Y}_{n}(K \Theta)\left[(K \Theta)^{2} / 2-n(n+1)+\mathrm{N}_{n}(K \Theta)\right], \\
a_{41}=-n \mathrm{~J}_{n}(L)\left[\mathrm{M}_{n}(L)-(n+1)\right], & a_{42}=\mathrm{J}_{n}(L \Theta)\left[(L \Theta)^{2} / 2-n(n+1)+\mathrm{M}_{n}(L \Theta)\right], \\
a_{43}=-n \mathrm{Y}_{n}(L)\left[\mathrm{N}_{n}(L)-(n+1)\right], & a_{44}=\mathrm{Y}_{n}(L \Theta)\left[(L \Theta)^{2} / 2-n(n+1)+\mathrm{N}_{n}(L \Theta)\right] .
\end{array}
$$

$K$ and $L$ are expressed in terms of plate velocity and $\Theta^{2}=2 /(1-\sigma)$. The various special solutions can, by making suitable approximations, be derived from equation (12). This is helpful in classifying the resonances. 
Typically, if $n=0$ the eigenfunctions are independent of angle and equation (12) degenerates into the product of two $2 \times 2$ determinants:

$$
f(K)=\left|\begin{array}{ll}
b_{11} & b_{13} \\
b_{21} & b_{23}
\end{array}\right| \times\left|\begin{array}{ll}
b_{32} & b_{34} \\
b_{42} & b_{44}
\end{array}\right|=0,
$$

where

$$
\begin{array}{ll}
b_{11}=\mathrm{J}_{1}(K)\left[\mathrm{M}_{1}(K)-(1-\sigma)\right], & b_{13}=\mathrm{Y}_{1}(K)\left[\mathrm{N}_{1}(K)-(1-\sigma)\right], \\
b_{21}=\mathrm{J}_{1}(L)\left[M_{1}(L)-(1-\sigma)\right], & b_{23}=\mathrm{Y}_{1}(L)\left[\mathrm{N}_{1}(L)-(1-\sigma)\right], \\
b_{32}=\mathrm{J}_{1}(K \Theta)\left[\mathrm{M}_{1}(K \Theta)-2\right], & b_{34}=\mathrm{Y}_{1}(K \Theta)\left[\mathrm{N}_{1}(K \Theta)-2\right], \\
b_{42}=\mathrm{J}_{1}(L \Theta)\left[\mathrm{M}_{1}(L \Theta)-2\right], & b_{44}=\mathrm{Y}_{1}(L \Theta)\left[\mathrm{N}_{1}(L \Theta)-2\right]
\end{array}
$$

The first determinant is for radial modes [6] and the other is for tangential modes [7].

All pure (other than $(1, R)$ ) and certain compound mode frequencies go to very high values as the ring becomes narrow $(\phi \rightarrow 1)$. By using the approximation that terms in $K$ and $L$ are large in comparison with all others, an equation which is the product of two $2 \times 2$ determinants, where the $c$ terms are the approximation of the $a$ equivalents, is obtained:

$$
\left|\begin{array}{ll}
c_{11} & c_{13} \\
c_{21} & c_{23}
\end{array}\right| \times\left|\begin{array}{ll}
c_{32} & c_{34} \\
c_{42} & c_{44}
\end{array}\right|=0
$$

where

$$
\begin{aligned}
& c_{11}=\mathrm{J}_{n}(K), \quad c_{13}=\mathrm{Y}_{n}(K), \quad c_{21}=\mathrm{J}_{n}(L), \quad c_{23}=\mathrm{Y}_{n}(L) \\
& c_{32}=\mathrm{J}_{n}(K \Theta), \quad c_{34}=\mathrm{Y}_{n}(K \Theta), \quad c_{42}=\mathrm{J}_{n}(L \Theta), \quad c_{44}=\mathrm{Y}_{n}(L \Theta)
\end{aligned}
$$

One determinant is for shear waves and the other for plate waves. Using the trigonometric approximations valid for high argument values of Bessel functions gives

$$
\sin K(1-\phi) \sin K \Theta(1-\phi)=0 .
$$

The elimination of $n$, the number of radial nodes, in the approximation means that the high frequency compound vibrations are effectively of pure plate or pure shear waves. This is understandable, as for large $K$ values the nodal circles are much closer together than the nodal radii. It will be noticed that the disk mode counterparts of the resonances cannot be identified from equation (13) or (14) and must be traced by following the frequency through successive reductions in $\phi$, using equation (12).

Holland's disk equation [3] is obtained by equating $b$, the hole radius, to zero. Equation (12) then simplifies to

$$
\left|\begin{array}{ll}
a_{11} & a_{12} \\
a_{31} & a_{32}
\end{array}\right|=0
$$

Numerical solutions were obtained by evaluating equation (12) for fixed values of $n, \phi$ and $\sigma$ with successive steps of $K$. This method was used to identify and follow the frequency variation of various modes as $\phi$ and $\sigma$ values were changed. The precise values shown in the various tables were obtained by interpolating between positive and negative values about the zero value of $f(K)$ by the Newton-Raphson method. As many hundred solutions were required, considerable effort was devoted to improving the calculation time. An average of about $\frac{1}{2}$ second per solution was achieved with an ICL 1905E computer. Solutions were obtained for values of $\sigma$ from 0 to 0.5 in steps of 0.01 . $\phi$ values from 0 to 0.9 in steps of 0.1 


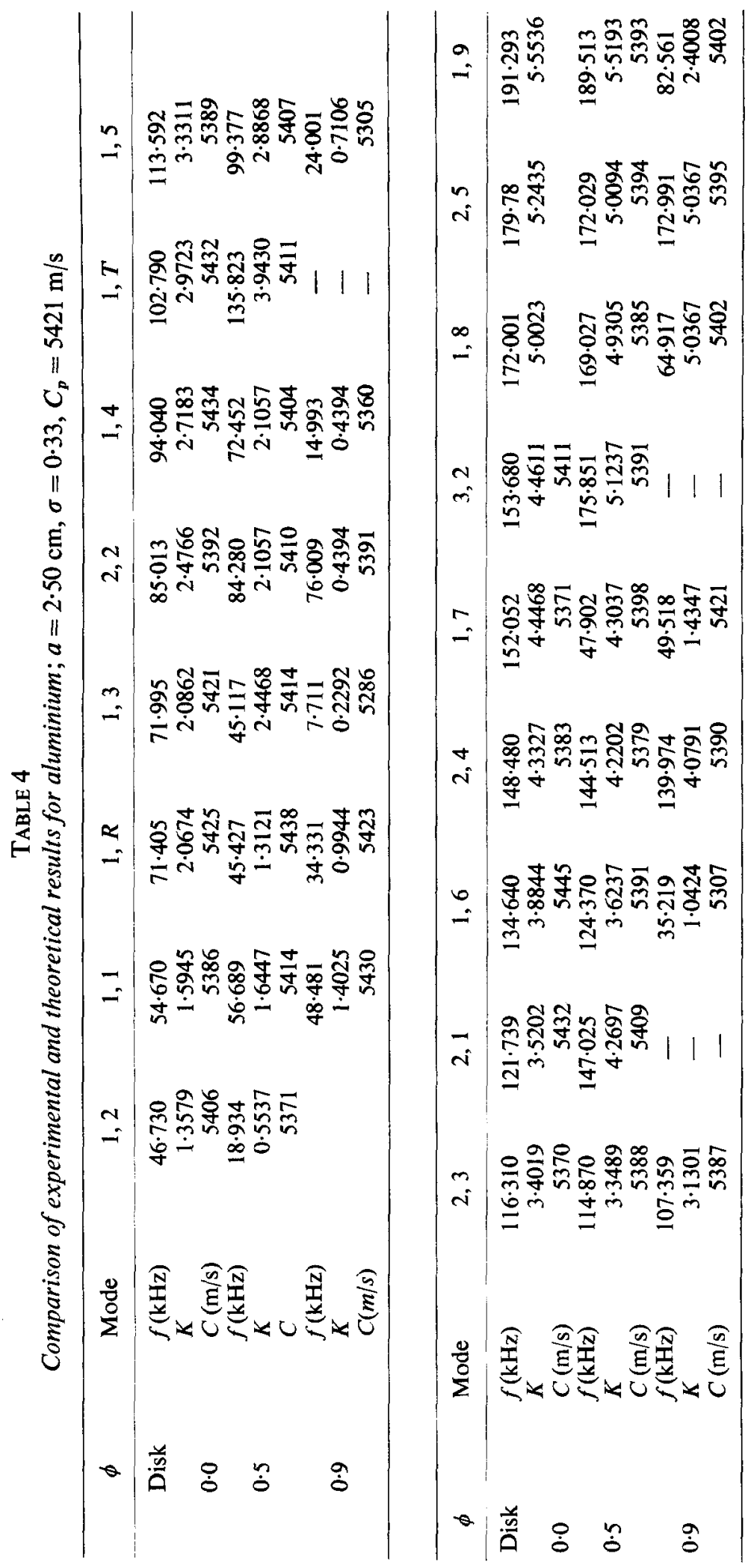


together with $\phi=0.99$ were used. The latter value was used in specific cases to establish the narrow ring asymptotic frequencies. Selected values are given in the various tables.

\section{EXPERIMENT}

Measurements by Onoe [8] and Holland [3] were restricted to piezoceramic materials only. The authors have made use of a method [9] applicable to any material [10]. Basically the resonant spectrum is obtained by driving the ring with a long thin wire fixed radially or at an angle to the periphery. A burst of longitudinal oscillations is transmitted up the wire, which must introduce sufficient delay to separate the signal returned from that transmitted. Under these conditions - the absence of standing waves-the wire presents a purely resistive load to the resonator which, while limiting the sharpness of resonance, has no effect on the resonant frequencies. The structure of the signal returned enables the resonant condition to be identified very precisely. The degree of coupling to a particular resonance depends on the direction of vibration at the point of drive, a radial drive giving good coupling when the radial component is large and an angled drive favouring shear modes. Where two frequencies are close together a double drive at different points can, by phasing, suppress one mode in favour of the other. The detailed vibration pattern across the face of a resonator was explored by a probe of fine wire used as a receiver. As a node is traversed the amplitude falls to zero and then rises with reverse phase.

Six resonators, progressing in form from a disk to a narrow ring, were cut from steel and aluminium rods about 2 inches in diameter. By using the drive techniques described, all the low order resonances could be obtained and, by the use of the probe and extrapolation from known frequency constants, identified. The lower of the high frequency modes such as $(2,1)$ and $(3,2)$ could be obtained for the disk and low $\phi$ value rings but became increasingly difficult to excite and identify with increase in hole size.

Selected resonances of the disks and annular rings for aluminium are shown in Table 4. By using the $(1, R)$ and $(1,3)$ disk frequencies the plate velocity and Poisson's ratio were calculated [10]. The $K$ values of the modes are available in steps of 0.01 and the series nearest the value calculated-in this case 0.33 - were used in the table. A value of plate velocity can be obtained from each frequency and the corresponding $K$ value. The constancy of the values shows the agreement between experiment and theory. A variety of lines and drive configurations was needed to obtain the full spectrum, the frequencies ranging from less than $10 \mathrm{kHz}$ to over $150 \mathrm{kHz}$. Departures from nominal velocity values are in most cases well under $1 \%$ and are attributed to the accumulated experimental errors arising from using different line drives, the tuning of the magnetostrictive launchers and ambient temperatures changes over the period of the experiments.

TABLE 5

Correspondence of disk and ring modes

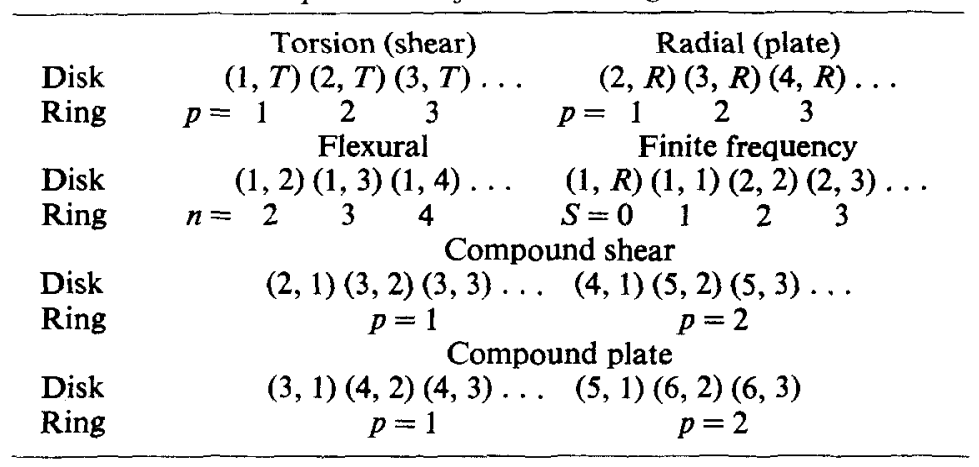


The experiments are considered to confirm the flexural and finite frequency modes over the whole range of hole size. In the case of other resonance series the agreement covered a sufficient range of hole size to give complete confidence in the theoretical results beyond the range of direct experiment.

\section{DISCUSSION OF RESULTS}

A complete picture summarized in Table 5 is now available of the ways in which disk and ring resonances constitute the limiting cases of annular ring resonances.

The pure torsion and pure radial modes are solutions of wave equations (10) and (11), respectively, where the associated velocities are those of shear and plate waves. The frequency increases rapidly with hole size, the nodal circles are crowded together, and the energy arising from the curvature becomes less and less. As a result the edges of the ring become antinodes and the width of the ring, $a(1-\phi)$, an integral number of half wavelengths, the integer being the number of nodal circles. Putting $a(1-\phi)=p \lambda / 2$ gives equation (17) expressing the phase velocity $C_{\text {phase }}$ in terms of $C$, the velocity associated with the particular $K$ term which is shear for torsion and plate for radial modes:

$$
C_{\text {phase }} / C=K(1-\phi) / p \pi \text {. }
$$

$(1, T)$ and $(2, R)$, each having one nodal circle, are the lowest modes of their respective series. Figures 1 and 2, giving $C_{\text {phase }} / C$, show the ways in which the shear and plate velocities are approached as $\phi$ increases.

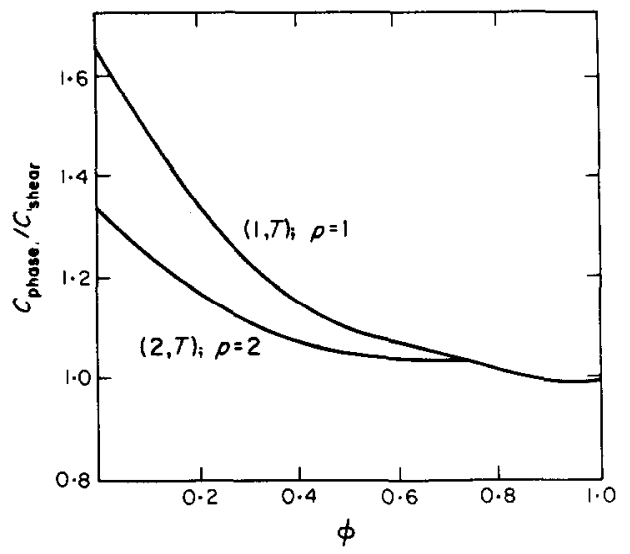

Figure 1. In pure torsional modes the wave velocity approaches that of shear waves for the narrow ring. This velocity has no dependence on $\sigma$ other than as a shear velocity.

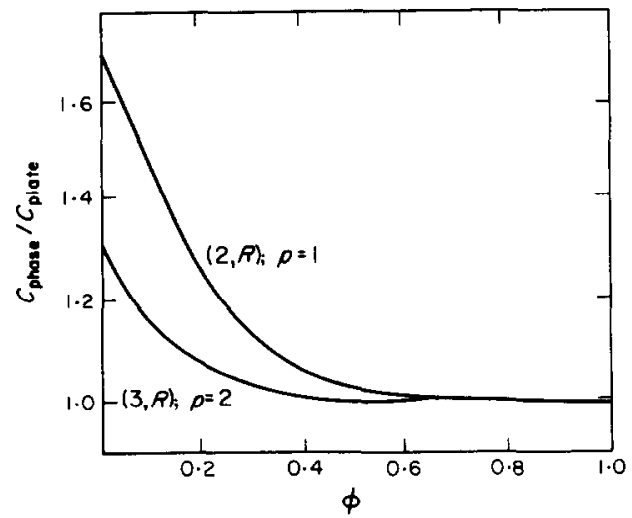

Figure 2. The first two pure radial modes for steel $(\sigma=0 \cdot 3), p=1$ and 2 respectively. The velocity has a small dependence on $\sigma$ outside the plate velocity term. 


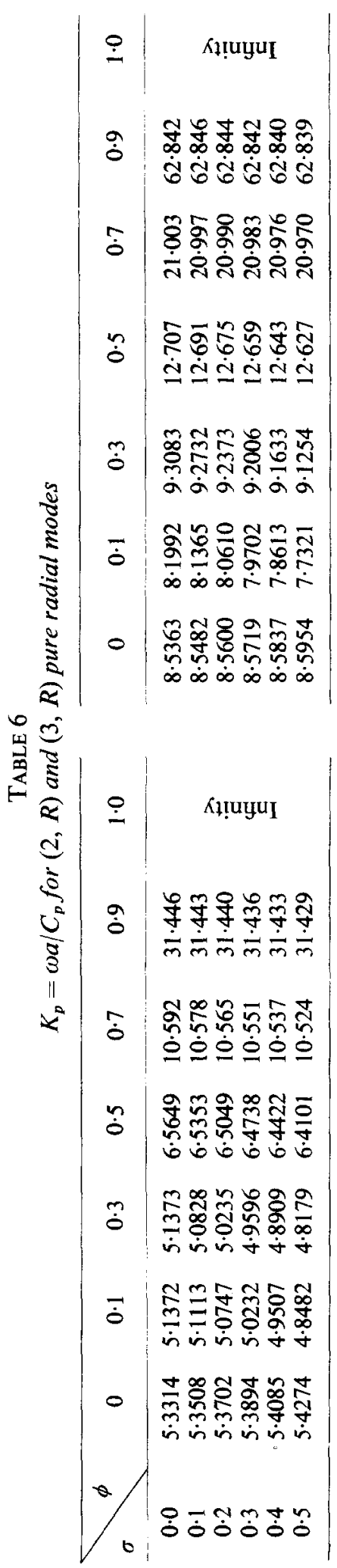


It will be seen that in both cases $C_{\text {phase }} / C$ goes to unity for all modes, establishing that the waves travel with the velocities appropriate to the wave equations and are not dispersive. The curves are not of a particularly regular form and the plateau which occurs in Figure 1 is a feature of similar perturbations in other modes. In the case of the velocity ratios, for torsional modes there is no dependence on $\sigma$ but this is not true for radial waves (Figures 1 and 2 and Table 6 ).

The compound modes of disks which have nodal diameters but no nodal circles $(1, n)$ are the direct counterpart of the flexural modes of rings. Figure 3 shows the progression for steel from the finite frequency disk resonances to those of the zero frequency thin ring. Table 7 gives the $K_{0}$ values for the full range of $\sigma$ and $\phi$. As the narrow ring region is approached the frequency becomes less dependent on Poisson's ratio and the slope finally follows the Hoppe equation [11] of Table 3.

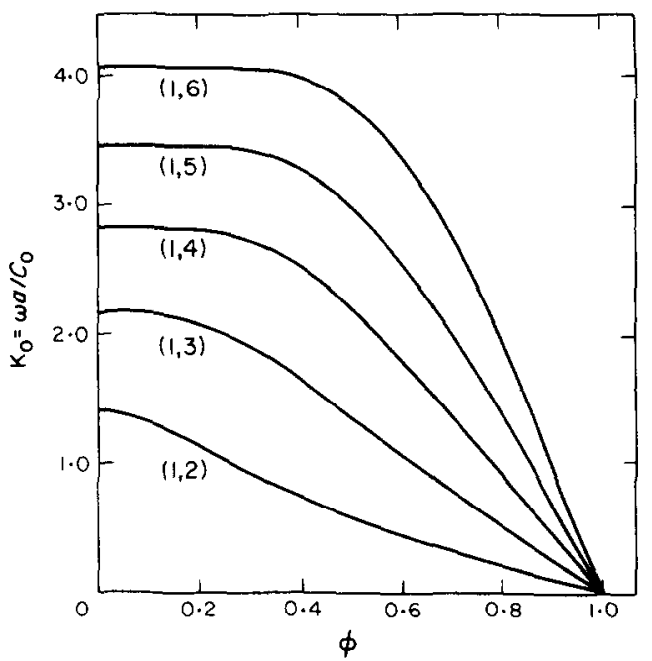

Figure 3. The flexural modes for steel $(\sigma=0 \cdot 3)$, showing the approaches to zero frequency for narrow rings. These modes have no nodal circles.

The finite frequency ring modes and their disk counterparts are of particular interest. It is reasonably evident that the $(1, R)$ disk mode becomes the $S=0$ ring mode but the disk counterparts of the higher $S$ value modes are not self-evident. In early experiments using radial drive the transformations from $S=0$ to $(1, R)$ and $S=1$ to $(1,1)$ were readily followed. Higher modes required drives with a tangential component. This extensional character of the strain results in large hoop stresses whereas in the distortion modes the stress is mainly flexural. The eigenfunctions of the ring [2] show $S$ to be the ratio of tangential to radial displacement and in disks a similar feature appears. The experiments established that the $S=2,3, \ldots, S$ ring modes have the disk counterparts of $(2,2),(2,3), \ldots,(2, n)$. For disks there is one nodal circle, but as the hole size increases it remains in the same position. Consequently, as the inner boundary pierces the node the vibrational amplitude goes to zero, reverses in phase and the inner and outer boundary amplitudes finally become equal as the extensional ring condition is reached. The results for steel are shown in Figure 4 and the complete data in Table 8 . With the exception of that for $(1, R)$ the curves have similar undulations which are thought to arise from the nodal circle effect described above. The $(1, R)$ disk frequency is greater than that of the $(1,1)$ mode and the curves cross at a low $\phi$ value as the frequencies move to the $K_{0}=1$ and $K_{0}=\sqrt{ } 2$ ring values. The cross-over occurs for all values of $\sigma$, the positions being $\phi=0.372$ and 0.143 for $\sigma=0.5$ and 0.0 , respectively. 


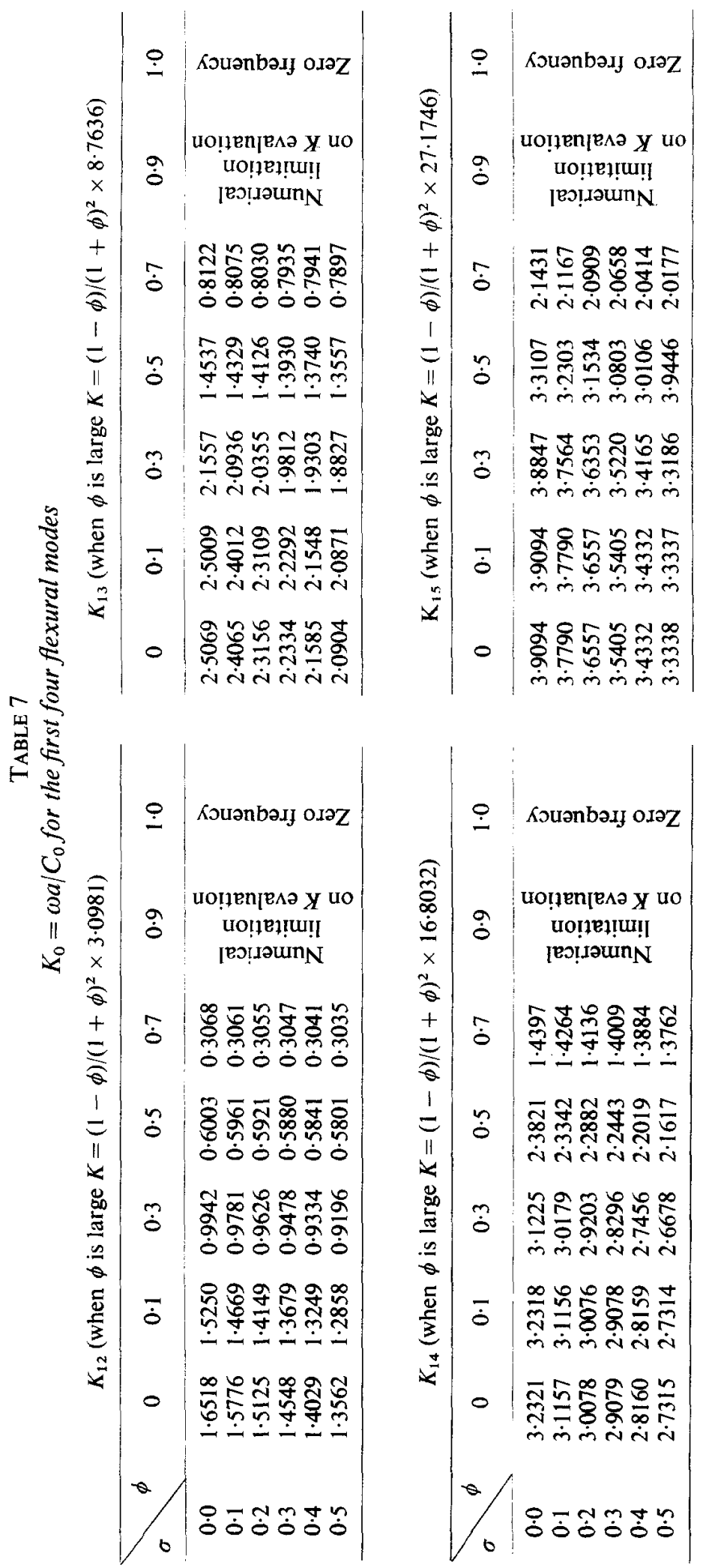



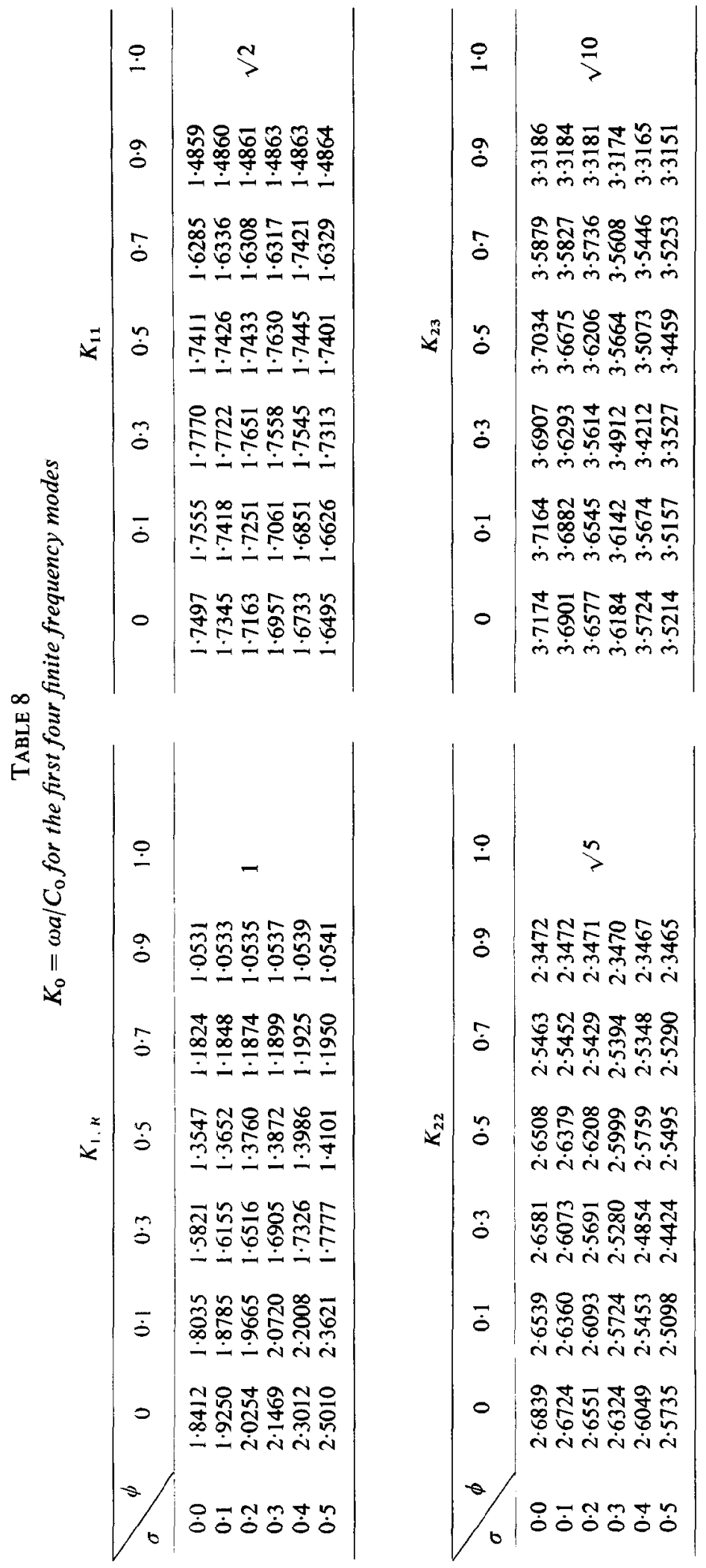


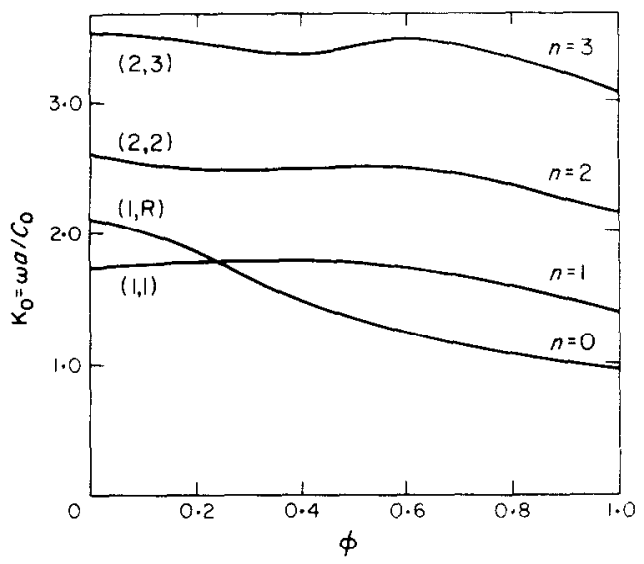

Figure 4. In the finite frequency modes the dependence of frequency on Poisson's ratio falls to zero in the narrow ring condition, the values being in the ratios $1: \sqrt{2}: \sqrt{5}: \sqrt{10}$. The movement is purely radial for the first mode and becomes increasing tangential for higher modes.

There remain those compound modes which, like pure torsional and plate modes, approach infinite frequency as $\phi \rightarrow 1$, the asymptotic values being the solutions of equation (13). These solutions, where $K$ refers to plate or shear waves and $p$ is the number of nodal circles, are given by

$$
K(1-\phi)=\pi p
$$

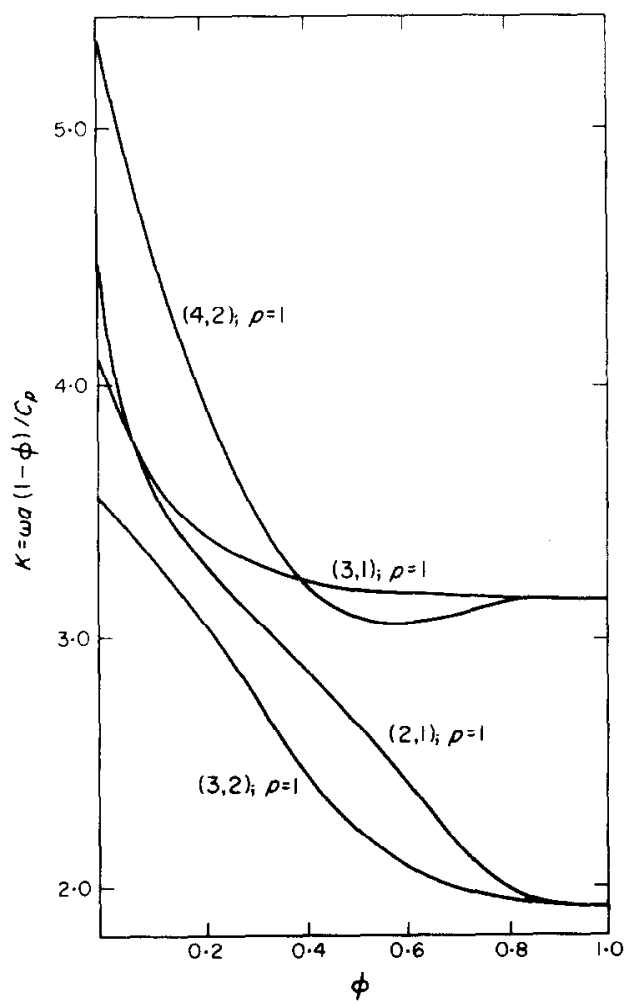

Figure 5 . The $K_{p}(1-\phi)$ values for the $(3,1)$ and $(4,2)$ plate modes and the $(2,1)$ and $(3,2)$ shear modes plotted as a function of $\phi$. The asymptotic values are $\pi$ and $\pi C_{s} / \pi C_{p}$ establishing the compound shear and plate mode series. 


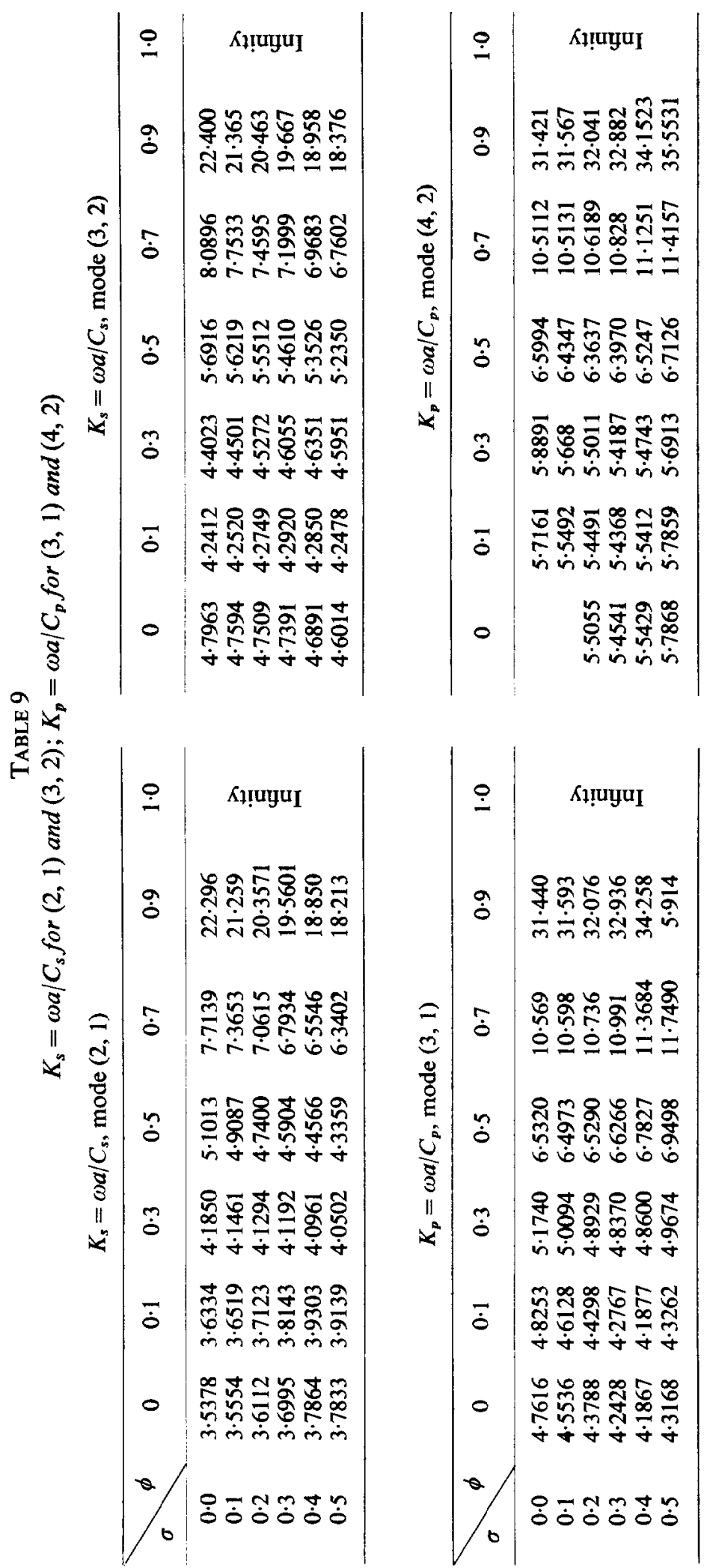


TABLE 10

Wave velocity ratios for compound shear and plate modes for a narrow ring $(\phi=0.99)$

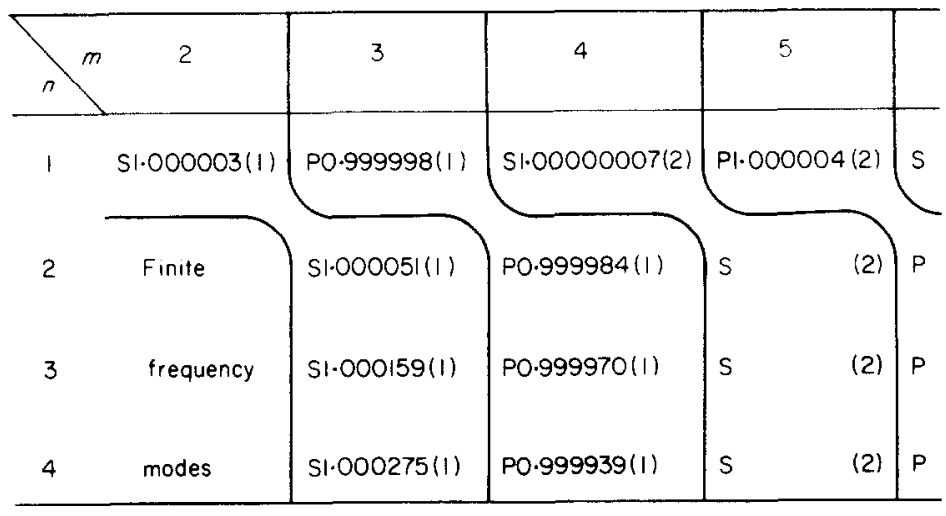

The way in which this limit is approached is shown for two plate modes- $(3,1)$ and $(4,2)-$ and for two shear modes- $(2,1)$ and $(3,2)$ - in Figure 5. Values of $K_{p}$ were obtained by solving equation (12) for $\sigma=0.25$ for all four modes and $K_{p}(1-\phi)$ is plotted against $\phi$. For the two plate modes the asymptotic value is $\pi$ and for the shear modes $\pi / 1 \cdot 633$. The ratio of $C_{p} / C_{s}$ is 1.633 for $\sigma=0.25$ giving a $K_{s}(1-\phi)$ value of $\pi . p$, the number of nodal circles, is therefore unity for all four modes. The eigenvalues for these four modes are given in Table 9.

Other modes were investigated for $\phi=0.99$ and $\sigma=0.25$, and the results are summarized in Table 10, which gives the $C_{\text {phase }} / C$ values of equation (17). The original disk modes are identified by the $(m, n)$ values. The lowest mode is the $(2,1)$ which heads the series $(2,1)$, $(3,2),(3,3), \ldots$, and higher series are $(3,1),(4,2),(4,3), \ldots$, and so on. The prefix letter gives the wave type and the suffix number the $p$ value. Thus the $(5,1)$ disk mode with four nodal circles becomes the $p=2$ narrow plate mode, two circles having been lost. The departure of the wave velocity ratio from unity is very small. It changes slightly with the number of nodal diameters and was found to have a similar small dependence on Poisson's ratio. Table 11 shows the actual progression of $K_{p}(1-\phi)$ solutions of equation (12). The disk solutions are in the sequence $(2,1),(3,1),(4,1), \ldots$, but, because of the difference between shear and plate wave velocities, the sequence is different for narrow rings. For example the $(5,1)$, a plate mode, has a higher frequency than the $(6,1)$, a shear mode.

The disappearance of nodal circles as the hole is developed in the disk occurs in the cases of the $(2,1)$ and higher finite frequency modes and the higher compound modes. The phenomenon was observed in detail for the $(2,1)$ mode by using rings of increasing hole size and investigating the phase and amplitude at the ring boundaries with a pick-up probe. The nodal circle

TABLE 11

$K_{p}=\omega a / C_{p}$ for the lower order compound modes. Narrow ring; $K_{p}(1-\phi), \phi=0.99$

\begin{tabular}{|c|c|c|c|c|}
\hline $\begin{array}{c}\text { Nodal circles } \\
(p)\end{array}$ & 1 & 2 & 3 & 4 \\
\hline Plate & & & & $2.57(9,1)$ \\
\hline Shear & $1.92(2,1)$ & 51 & 776 & $.70(8,1)$ \\
\hline
\end{tabular}


did not change position significantly with hole size and disappeared as already described in the discussion of Figure 4 and Table 8. There is an apparent paradox, as for a particular hole size the inner boundary can be a node, meaning in this case that there is no radial movement $\left(\xi_{r}=0\right)$. In terms of the boundary condition given in equation (10), the radial stress associated with the $\partial \xi_{r} / \partial r$ term is cancelled out by the $(\sigma / r) \partial \xi_{\theta} / \partial \theta$ term which produces the hoop stress. There is thus no net radial force and therefore no radial movement.

There is a close relationship between the thin ring modes and those of infinite hollow cylinders for the non-propagating case (the cut-off frequency condition). All thin ring modes have their counterparts in cylinders which have two additional series, shear modes where the shear is in the longitudinal direction and longitudinal rod modes. The numerical results obtained by Armenakas et al. [5] for $\sigma=0.3$ are given in ascending order of frequency for successive orders $(n)$ of the Bessel function. Six values $\left(\Omega_{1}\right.$ to $\left.\Omega_{6}\right)$ are given for the first five $n$ values. Table 12 relates the $(\Omega, n)$ values to the disk classifications of Table 1 . The torsional and radial modes above the first radial are omitted.

TABLE 12

Identification of infinite shell modes by $(\Omega, n)$. The frequencies are in ascending order of $\Omega$ for a given $n$. Thus, the third shear $z$ overtone is at a lower frequency than the seconds of shear $\theta$ and bulk $r$

\begin{tabular}{lllll}
\hline Mode type & \multicolumn{4}{c}{$(\Omega, n)$ values } \\
\cline { 1 - 2 } Flexural & 1,2 & 1,3 & 1,4 & $\ldots$ \\
Finite $f$ & 2,0 & 3,1 & 3,2 & $\ldots$ \\
Shear $\theta 1$ & 5,1 & 5,2 & 5,3 & $\ldots$ \\
Shear $z 1$ & 3,0 & 4,1 & 4,2 & $\ldots$ \\
Shear $z 2$ & 5,0 & $\ldots$ & $\ldots$ & $\ldots$ \\
Shear $z 3$ & 6,0 & $\ldots$ & $\ldots$ & $\ldots$ \\
Bulk $r 1$ & 4,0 & 6,1 & 6,2 & $\ldots$ \\
Rod $z n$ & 2,1 & 2,2 & 2,3 & $\ldots$ \\
\hline
\end{tabular}

The pure torsional modes (Figure 1) have the same frequencies and these are independent of Poisson's ratio except insofar as it appears in the expression for the shear velocity. For zero Poisson's ratio the rod, plate and dilatational (bulk) velocities are equal and all the ring and shell frequencies are equal. With increase in Poisson's ratio the frequencies diverge and for some modes reach infinity at $\sigma=0.5$ where the dilatational velocity is infinite. For the thin shell and narrow ring the Rayleigh and Hoppe mode (see Table 3 ) frequencies are in the ratio of plate to rod velocities and those of the cylinder and disk radial modes are in the ratios of dilatational to plate velocities.

To summarize, every disk mode has been followed to its narrow ring counterpart, which can approach zero, finite or infinite frequency. The general solution of the annulus given in equation (12) contains the Rayleigh [2] and Hoppe [1] ring resonances and the Poisson [11], Love [4] and Holland [3] disk resonances. Five figure tables of frequency eigenvalues for a full range of annulus geometry and Poisson's ratio are available.

\section{ACKNOWLEDGMENTS}

The authors wish to thank the Science Research Council (JCKS) and the University of Aston (GA) for studentships, Mr A. E. Sutton for his assistance with various mathematical problems and Mr F. Hunt for preparing the large number of precision specimens needed. 


\section{REFERENCES}

1. R. Hoppe 1871 Journal für Mathematik (Crelle) 73, 158-168. Vibrationen eines Ringes in seiner Ebene.

2. LORD RAYLEIGH 1889 Proceedings of the London Mathematical Society 20, 225-234. On the free vibrations of an infinite plate of homogeneous isotropic elastic matter.

3. R. Holland 1966 Journal of the Acoustical Society of America 40, 1051-1057. Numerical studies of elastic disk contour modes lacking axial symmetry.

4. A. E. H. Love 1944 A Treatise on the Mathematical Theory of Elasticity. New York: Dover Press.

5. A. E. Armenakas, D. C. Gazis and G. Herrmann 1969 Free Vibrations of Circular Cylindrical Shells. London: Pergamon Press.

6. C. V. STEPHENSON 1966 Journal of the Acoustical Society of America 28, 51-56. Radial vibrations in short hollow cylinders of barium titanate.

7. D. C. GAzIS 1958 Journal of the Acoustical Society of America 30, 786-794. Exact analysis of plane strain thick walled hollow cylinders.

8. M. ONOE 1956 Journal of the Acoustical Society of America 28, 1158-1162. Contour vibrations of isotropic plates.

9. J. F. W. BELl, A. C. JoHNSON and J. C. K. SHARP 1975 Journal of the Acoustical Society of America 57, 1085-1093. A pulse-echo method of investigating the properties of mechanical resonators.

10. J. F. W. BELL and J. C. K. ShARP 1974 Revue International des Hautes Temperatures et Réfractoires 12, 40-43. The precision measurements of the elastic constants of isotropic solids over a wide temperature range.

11. S. D. Polsson 1829 Memoire de l'Academie de Paris 8. Memoire sur l'equilibre et le movement des corps élastique.

\section{APPENDIX: LIST OF SYMBOLS}

$\begin{array}{ll}a & \text { disk radius }(\mathrm{m}) \\ b & \text { hole radius }(\mathrm{m}) \\ a_{i j}, b_{l j}, c_{l j} & \text { terms in determinant } \\ A_{l} & \text { amplitude coefficients } \\ C & \text { a velocity of sound }(\mathrm{m} / \mathrm{s}) \\ C_{0}^{2} & E / \rho, \text { rod }(\mathrm{m} / \mathrm{s})^{2} \\ C_{p}^{2} & C_{0}^{2} /\left(1-\sigma^{2}\right), \text { plate }(\mathrm{m} / \mathrm{s})^{2} \\ C_{s}^{2} & C_{0}^{2} / 2(1+\sigma), \text { shear } / \text { torsion }(\mathrm{m} / \mathrm{s})^{2} \\ E & {\text { Young's modulus }\left(\mathrm{N} / \mathrm{m}^{2}\right)} \\ M_{n}(x), \mathrm{N}_{n}(x) & x \mathrm{~J}_{n-1}(x) / \mathrm{J}_{n}(x), \text { etc., Onoe's modified Bessel quotients } \\ J_{n}, Y_{n} & \text { Bessel functions of } 1 \mathrm{st} \text { and } 2 \text { nd kind } \\ L & h b \\ h & \text { o/ } / C_{p} \text { plate wave number }\left(\mathrm{m}^{-1}\right) \\ k & \omega / C_{s} \text { shear wave number }\left(\mathrm{m}^{-1}\right) \\ K & \omega a / C, \text { a dimensionless frequency constant } \\ (m, n) & \text { integers defining a disk mode } \\ n & \text { integers defining ring distortional mode } \\ p & \text { integer }(\text { equation }(17)) \\ r, \theta & \text { cylindrical co-ordinates } \\ s & \text { integer defining ring extensional mode } \\ S & \text { strain } \\ T & \text { stress }\left(\mathrm{N} / \mathrm{m}^{2}\right) \\ \xi & \text { a displacement }(\mathrm{m}) \\ A & \text { dilational strain } \\ \phi & \text { b/a } \\ \rho & \text { density }\left(\mathrm{kg} / \mathrm{m}^{3}\right) \\ \sigma & \text { Poisson's ratio } \\ \bar{\omega} & \text { rotational strain } \\ \omega & \text { angular frequency }\left(\mathrm{s}^{-1}\right) \\ \Theta^{2} & 2 /(1-\sigma) \\ & \end{array}$

